\title{
Prevalence of Congenital Malaria in Minna, North Central Nigeria
}

\author{
Innocent Chukwuemeka James Omalu, ${ }^{1}$ Charles Mgbemena, ${ }^{2}$ \\ Amaka Mgbemena, ${ }^{1}$ Victoria Ayanwale, ${ }^{1}$ Israel Kayode Olayemi, ${ }^{1}$ \\ Adeniran Lateef, ${ }^{3}$ and Victoria I. Chukwuemeka ${ }^{1}$ \\ ${ }^{1}$ Department of Biological Sciences, Federal University of Technology, Minna, Nigeria \\ ${ }^{2}$ Dentistry Department, Niger State General Hospital, Minna 900002, Nigeria \\ ${ }^{3}$ Department of Biochemistry/Physiology, University of Abuja, FCT, Nigeria
}

Correspondence should be addressed to Innocent Chukwuemeka James Omalu, omalu_icj@hotmail.com

Received 14 April 2011; Revised 24 June 2011; Accepted 28 June 2011

Academic Editor: Demba Sarr

Copyright (c) 2012 Innocent Chukwuemeka James Omalu et al. This is an open access article distributed under the Creative Commons Attribution License, which permits unrestricted use, distribution, and reproduction in any medium, provided the original work is properly cited.

\begin{abstract}
The study was designed to determine the true prevalence of congenital, cord, and placental malaria in General Hospital Minna, North Central Nigeria. Peripheral blood smears of near-term pregnant women, as well as the placental, cord, and peripheral blood smears of their newborn babies, were examined for malaria parasites, using the Giemsa staining technique. Out of 152 pregnant women screened, 21 (13.82\%) of them were infected with malaria parasites. Of the 152 new born babies, $4(2.63 \%)$ showed positive peripheral parasitaemia. Placental parasitaemia was 7/152 (4.61\%), while cord blood parasitaemia was 9/152 (5.92\%). There were strong associations between peripheral and cord malaria parasitaemia and congenital malaria $(P<0.05)$. Plasmodium falciparum occurred in all, and none had mixed infection. The average birth weights of the babies delivered of nonmalarious pregnant women were higher than those delivered by malarious pregnant women, though not significant $(P>0.05)$. Malaria parasitaemia occurred more frequently in primigravidae than multigravidae.
\end{abstract}

\section{Introduction}

Congenital malaria was first described in 1876 [1]. It can be acquired by transmission of parasites from mother to child during pregnancy or perinatally during labour [2].

Congenital malaria has been documented for many years, but it was previously thought to be uncommon especially in indigenous populations; more recent studies, however, suggest that incidence has increased, and values between 0.30 to $33.00 \%$ have been observed from both endemic and nonendemic areas [3].

Malaria and pregnancy are generally believed to be mutually aggravating conditions. The pathological changes due to malaria and the physiological changes associated with pregnancy have a synergistic effect on the course of each other [4].

Pregnancy exacerbates malaria through a nonspecific hormone-dependant depression of the immune system; protective antiplasmodial activity is suppressed at pregnancy [5].

The following hypotheses to explain the altered immunity associated with pregnancy were offered.
Reduced lymphoproliferative response sustained by elevated levels of serum cortisol, loss of cell-mediated immunity in the mother, the presence of placenta, a new organ in the primigravidae, allows the parasite to bypass the existing host immunity, or allows placenta-specific phenotypes of $P$. falciparum to multiply; pregnant women display a bias towards type- 2 cytokines and are therefore susceptible to diseases requiring type-1 responses for protection like TB, malaria, and $P$. falciparum has the unique ability of cytoadhesion; chondroitin sulfate A and hyaluronic acid are the adhesion molecules for parasite attachment to placental cells [4].

In pregnancies complicated by malaria, both fetal growth retardation and preterm birth contribute to low birth weight [6]. Malaria control still remains the foremost public health challenge in Africa, and world malaria report, which indicated that Nigeria accounts for a quarter of all malaria cases in the 45 malaria endemic countries in Africa, clearly showed the enormity of the socioeconomic and health burdens of the disease in the country, even in the face of dearth of 
TABle 1: Prevalence of Plasmodium falciparum in blood samples of different groups of women and tissues at General Hospital Minna, North Central Nigeria.

\begin{tabular}{|c|c|c|c|c|c|c|c|c|c|}
\hline \multirow{2}{*}{ Source of blood } & \multicolumn{3}{|c|}{ Overall } & \multicolumn{3}{|c|}{ Primigravidae } & \multicolumn{3}{|c|}{ Multigravidae } \\
\hline & No examd & $\begin{array}{c}\text { No } \\
\text { positive }\end{array}$ & $\begin{array}{c}\text { Infect rate } \\
(\%)\end{array}$ & No examd & $\begin{array}{c}\text { No } \\
\text { positive }\end{array}$ & $\begin{array}{c}\text { Infect rate } \\
(\%)\end{array}$ & No examd & $\begin{array}{c}\text { No } \\
\text { positive }\end{array}$ & $\begin{array}{c}\text { Infect rate } \\
(\%)\end{array}$ \\
\hline $\begin{array}{l}\text { Near-term } \\
\text { Pregnant women }\end{array}$ & 152 & 21 & 13.82 & 76 & 12 & 15.79 & 76 & 9 & 11.84 \\
\hline $\begin{array}{l}\text { Nonpregn } \\
\text { women }\end{array}$ & 100 & 13 & 13.00 & - & - & - & - & - & - \\
\hline Neonate & 152 & 4 & 2.63 & - & 2 & - & - & 2 & - \\
\hline Placenta & 152 & 7 & 4.61 & 76 & 3 & 3.95 & 76 & 4 & 5.26 \\
\hline Cord & 152 & 9 & 5.92 & 76 & 4 & 5.26 & 76 & 5 & 6.58 \\
\hline Total & 708 & 54 & 7.63 & 226 & 21 & 9.29 & 228 & 20 & 8.77 \\
\hline
\end{tabular}

information on the epidemiology of the disease. This, thus, informed this study on the prevalence of congenital malaria in Minna, an often neglected but germane aspect of the epidemiology of malaria in endemic communities [7].

\section{Materials and Methods}

2.1. Study Site and Population. The study was carried out at General Hospital Minna, North Central Nigeria. Minna, the capital of Niger State, Nigeria, is located within longitude $6^{\circ} 33^{\prime} \mathrm{E}$ and latitude $9^{\circ} 37^{\prime} \mathrm{N}$, covering a land area of $88 \mathrm{~km}^{2}$ with a population of 1.2 million. Minna has a tropical climate with mean annual temperature, relative humidity, and rainfall of $30.20^{\circ}, 61.00 \%$, and $1334.00 \mathrm{~cm}$, respectively. The climate presents two distinct seasons: a rainy season (April-October) and dry season (November-March). Minna is an endemic area for malaria.

The study was conducted from October 2010 to February 2011. The subjects were near-term (close to delivery) pregnant women who were delivered of their babies at the hospital. Sample sizes were determined from the number of pregnant women that attended antenatal care during the period of study. The nonpregnant women served as control to help compare malaria prevalence in pregnancy only. The pregnant women did not take any chemoprophylaxis and did not use mosquito treated nets for protection. The HIV status of the subjects were not determined.

2.2. Ethical Considerations. All work was performed according to the guidelines for human experimentations in clinical research stated by the Federal Ministry of Health of Nigeria. This study was approved by the ethical committee of General Hospital Minna, Nigeria. All women gave oral informed consent.

2.3. Sample Collections. Blood samples were obtained from the peripheral blood of 152 near-term pregnant women using sterile syringes. After delivery, blood was collected from the placentae by placental biopsies. The placentae were incised between the maternal and foetal surface and a small quantity of blood pipette out from the intervillous with a sterile syringe. Cord blood was collected immediately after separation of placenta, and peripheral blood of neonates was collected by heel pricking using a sterile blood lancet on clean glass slides. Labelled ethylene-diamine tetraacetic acid (EDTA) bottles were used in collecting blood samples for parasitological examinations. Blood collections were made possible with the assistance of midwives on duty during child delivery. Blood samples were also collected from 100 nonpregnant women.

The ages, types of birth, and weights of newborn babies were recorded.

2.4. Parasitological Examination. Thick and thin blood films were stained with $10 \%$ Giemsa and read for malaria parasites by two trained microscopists following standard quality control procedure. Parasitaemia was expressed as the number of asexual forms of $P$. falciparum per microlitre; a result was considered negative after a reading of 1000 leucocytes in the microscope $(\times 1000)$. Parasitaemia was graded as low (1$999 / \mu \mathrm{L})$, moderate $(1000-9999 / \mu \mathrm{L})$, and high $(>10000 / \mu \mathrm{L})$. Transplacental passage of Plasmodium falciparum was confirmed by detection of malaria parasite within 7 days of birth.

2.5. Statistical Analysis. All data were analysed using SPSS version 10.1 for windows. Descriptive statistics were computed for all relevant data. Chi square analysis was used to compare proportions within and among groups, for statistical significance.

\section{Results}

The prevalence of malaria infection in blood smears of nearterm pregnant mothers, their newborns, cords, placentae, and nonpregnant women was shown in Table 1. Out of a total of 152 near-term pregnant women screened, 21 (13.82\%) had parasite in their peripheral blood. Among 100 nonpregnant women screened, $13(13.00 \%)$ had peripheral malaria parasite. Infection rate was higher in pregnant women but not significant $(P>0.05)$ using the chi-square. The inclusion of nonpregnant women helped to compare the prevalence of malaria. Also, there was no significant difference in infection rates between the primigravidae $(15.79 \%)$ and the multigravidae (11.84\%), $(P>0.05)$. 
TAble 2: Average parasite densities (Parasites/uL) of blood of different groups of women and tissues at General Hospital Minna, North central Nigeria.

\begin{tabular}{lccccccc}
\hline Parasite densities & Pregnant women & Nonpregnant women & Newborn & Placenta & Cord & PG & MG \\
\hline Parasites/uL & 1415 & 411 & 128 & 689 & 196 & 1281 & 134 \\
\hline
\end{tabular}

PG: Neonate with primigravid mothers,

MG: Neonate with multigravid mothers.

TABle 3: Erythrocytic stages of Plasmodium falciparum in blood samples of different groups of women and tissues at General Hospital Minna, North central Nigeria.

\begin{tabular}{|c|c|c|c|c|c|c|c|c|c|c|c|c|c|c|}
\hline \multirow{2}{*}{ Source of blood } & \multirow{2}{*}{ No positive } & \multicolumn{2}{|l|}{ TR } & \multicolumn{2}{|l|}{ GM } & \multicolumn{2}{|l|}{$\mathrm{SCH}$} & \multicolumn{2}{|c|}{ TR \& SCH } & \multicolumn{2}{|c|}{ TR \& GM } & \multicolumn{2}{|c|}{ TR \& GM \& SCH } & \multirow[t]{2}{*}{ Total } \\
\hline & & No & $\%$ & No & $\%$ & No & $\%$ & No & $\%$ & No & $\%$ & No & $\%$ & \\
\hline Pregnant women & 21 & 60 & 64.50 & 25 & 26.80 & - & - & - & - & 8 & 8.60 & - & - & 93 \\
\hline Nonpreg women & 13 & 15 & 46.80 & 12 & 37.50 & - & - & - & - & 5 & 15.60 & - & - & 32 \\
\hline Newborn & 4 & 5 & 45.50 & 5 & 45.50 & - & - & - & - & 1 & 9.00 & - & - & 11 \\
\hline Placenta & 7 & 20 & 62.50 & 10 & 31.20 & - & - & - & - & 2 & 6.30 & - & - & 32 \\
\hline Cord & 9 & 12 & 36.30 & 10 & 30.30 & - & 一 & 一 & - & 3 & 9.00 & - & - & 33 \\
\hline Total & 54 & 112 & 62 & & & & & & & 19 & & & & 201 \\
\hline
\end{tabular}

TR: trophozoites, GM: gametocytes, $\mathrm{SCH}$ : schizonts.

Of the 152 newborn babies screened, 4 (2.63\%) had positive peripheral parasitaemia. Examination of the 152 placentae gave a prevalence of $4.63 \%$ (7) for malaria parasite, out of which 4 placentae were for the 4 babies with positive peripheral parasitaemia and known showed any clinical symptom. The only malaria parasite encountered in this study was the Plasmodium falciparum.

The prevalence of cord blood parasitaemia was 5.92\% (9/152), 5 of which came from multigravidae (6.58\%) and 4 from primigravidae (5.26\%). The average parasite density was $1,415 \mathrm{p} / \mathrm{uL}$ for pregnant women which was significantly $(P<0.05)$ higher than the $411 \mathrm{p} / \mathrm{uL}$ recorded for nonpregnant women. Average parasite density for newborns was $128 \mathrm{p} / \mathrm{uL}$, slightly lower than that for the cords which was $196 \mathrm{p} / \mathrm{uL}$; however, both were significantly lower than parasite density recorded in the placentae $($ mean $=689 \mathrm{p} / \mu \mathrm{L})$. Parasite density was significantly $(P<0.05)$ higher in neonates from primigravid $(1281 / \mathrm{uL})$ mothers than multigravids (134/uL) (Table 2). Parasite stages encountered were dominated by trophozoites (64.50\%), distantly followed by gametocytes $(26.80 \%)$ in pregnant women; the same trend was observed for nonpregnant women, placenta, cord, and neonates (Table 3 ).

The 4 parasitized newborns all had birth weights above $3 \mathrm{~kg}$. On the average, however, the birth weights of babies from parasitized mothers were lower than those from nonparasitized mothers, albeit, the difference was not significant $(P>0.05)$. Also, newborns from primigravid mothers weighed averagely less than those from multigravid mothers; again the difference was not significant (Table 4).

\section{Discussion}

Many researchers have reported high prevalence of malaria in pregnancy in different parts of Nigeria, ranging from $19.70 \%$ to $72.00 \%[8-11]$. The prevalence of malaria in pregnant women in this study was $14 \%$; though consistent with the reported Nigerian situation, the relatively low percentage could be due to the fact that the study was carried out during the dry season, a period of low mosquito density and, perhaps, low level malaria transmission rates. This observation supports the position that in areas of malaria endemicity, pregnancy is associated with increased susceptibility to malaria, arising from pregnancy-induced altered immunity [6], Immunosuppression from raised serum cortisol, loss of cell-mediated immunity, effects of a new organ, the placenta, and loss of type- 1 cytokine responses [4].

The prevalence of malaria in nonpregnant controls in this study was $13 \%$, although this was lower than the pregnant subjects; the difference was not significant. The inclusion of nonpregnant women in the study allows comparison of malaria prevalence in pregnancy with nonpregnant female subjects. Parasite densities for pregnant women were comparatively higher than that of nonpregnant women. This is consistent with the findings of Agomo et al. [8], in a study done in Lagos, Nigeria. Although none of the pregnant women screened took malaria chemoprophylaxis during pregnancy, nor used any form of mosquito nets, the parasite density figures were low. However, one may conclude that this may have to do with the period of the study, October to February, that is, early to mid-dry season. A season that coincides with decreasing densities of female Anopheles species and therefore reduced inoculation with parasites.

Low parasites densities were also recorded for the placentae, cords, and peripheral blood of newborns.

Each placentae of the 4 parasitaemia neonates in this study also had positive parasitaemia. This agrees with the position of Chedraui et al. [6], that placental infection is a prerequisite for, but does not predict, congenital malaria. A strong correlation between placental and congenital parasitaemia has been shown [12]. 
TABLE 4: Mean $( \pm \mathrm{SD})$ birthweights $(\mathrm{kg})$ of neonate of pregnant women at General Hospital Minna, North Central Nigeria.

\begin{tabular}{ccccc}
\hline Pregnant women & \multicolumn{2}{c}{ Primigravidae } & \multicolumn{2}{c}{ Multigravidae } \\
& Male & Female & Male & $3.18 \pm 0.31$ \\
Positive pregnant women & $3.03 \pm 0.5$ & $2.83 \pm 0.44$ & $3.31 \pm 0.14$ & \\
Negative pregnant women & $3.01 \pm 0.52$ & $2.89 \pm 0.25$ & $2.94 \pm 0.14$ \\
\hline
\end{tabular}

However, an increasing trend in prevalence of congenital malaria has been reported recently. In a multicentre study done at Ibadan a prevalence of $5.10 \%$ was reported in University College Hospital [13]. A prevalence of $46.70 \%$ was reported in a study of 120 newborn babies at Ile-Ife, Southwestern Nigeria [14]. Also a prevalence of $13.00 \%$ was reported among 546 in-born neonates at Calabar Teaching Hospital [15]. These findings represent a new trend since parasitaemia in peripheral blood of newborns was considered rare in highly endemic areas.

Infection with Plasmodium falciparum was the only one encountered in this study. This agrees with a similar study done in Libreville, Gabon by Bouyou-Akotet et al. [16], wherein only Plasmodium falciparum was encountered. It also agrees with widely accepted view that Plasmodium falciparum is the predominant species in Nigeria [16]. The predominant forms of this parasite seen were the trophozoites (ring stages).

The only stillbirth encountered in this study resulted from foetal distress. The mother of the baby was negative for malaria parasites (nonmalarious).

On the average, the birth weights of babies from parasitaemia mothers were lower than those of babies from nonparasitaemia mothers, but not significantly so. This disagrees with the widely held view that babies with parasitized placentae often have low birth weight. It also contradicts the findings of Adebami et al. [17], who stated that babies of mothers with parasitized placentae have mean birth weight significantly lower than babies of mothers with nonparasitized placentae. The generally low parasite densities found in this study may explain the minimal effect on birth weight, as observed by Opara [2] who recorded parasitized neonate twins with normal birth weights. Primigravidae are more susceptible to malaria infection than multigravidae in endemic areas [18]. The findings of this study supports this position, albeit, the difference in prevalence was not significant.

Congenital malaria was previously thought to be rare, especially, in areas of malaria endemicity. Though this study appears not to support this view, an increasing prevalence of congenital malaria among newborns in other areas of malaria endemicity has been observed.

\section{Conflict of Interests}

The authors declare that there is no conflict of interests.

\section{Acknowledgments}

The authors gratefully acknowledge the assistance of the technologists and midwives and for the use of laboratory facilities of General Hospital Minna and the Department of Biological Sciences, Federal University of Technology, Minna.

\section{References}

[1] S. Romand, P. Bouree, J. Gelez, B. Bader-Meunier, F. Bisaro, and J. P. Dommergues, "Congenital malaria. Infected twins born to an asymptomatic mother," Presse Medicale, vol. 23, no. 17, pp. 797-800, 1994.

[2] D. A. Opara, "Congenital malaria in newborn twins," Ghana Medical Journal, vol. 44, no. 2, pp. 76-78, 2010.

[3] S. A. Sotimehin, T. I. Runsewe-Abiodun, O. T. Oladapo, O. F. Njokanma, and D. M. Olanrewaju, "Possible risk factors for congenital malaria at a tertiary care hospital in Sagamu, Ogun State, South-West Nigeria," Journal of Tropical Pediatrics, vol. 54, no. 5, pp. 313-320, 2008.

[4] B. S. Kakkilaya, "Malaria and pregnancy," 2009, http://www .malariasite.com

[5] O. O. Okwa, "The status of malaria among pregnant women: a study in Lagos, Nigeria," African Journal of Reproductive Health, vol. 7, no. 3, pp. 77-83, 2003.

[6] P. A. Chedraui, J. Daily, B. Wylie, P. F. Weller, S. M. Ramin, and V. Barss, "Overview of malaria in pregnancy," 2009, http://www.uptodate.com.

[7] World Health Organisation, "World Malaria Report," WHO, Geneva, Switzerland, pp. 99-101, 2008.

[8] C. O. Agomo, W. A. Oyibo, R. I. Anorlu, and P. U. Agomo, "Prevalence of malaria in pregnant women in Lagos, SouthWest Nigeria," Korean Journal of Parasitology, vol. 47, no. 2, pp. 179-183, 2009.

[9] O. O. Okwa, "The status of malaria among pregnant women: a study in Lagos, Nigeria," African Journal of Reproductive Health, vol. 7, no. 3, pp. 77-83, 2003.

[10] M. B. Kagu, M. B. Kawuwa, and G. B. Gadzama, "Anaemia in pregnancy: a cross-sectional study of pregnant women in a Sahelian tertiary hospital in Northeastern Nigeria," Journal of Obstetrics and Gynaecology, vol. 27, no. 7, pp. 676-679, 2007.

[11] C. J. Uneke, F. E. Iyare, P. Oke, and D. D. Duhlinska, "Assessment of malaria in pregnancy using rapid diagnostic tests and its association with HIV infection and hematologic parameters in South-Eastern Nigeria," Haematologica, vol. 93, no. 1, pp. 143-144, 2008.

[12] O. A. Oduwole, G. C. Ejezie, and M. Meremekwu, "Congenital Malaria," American Journal of Tropical Medicine and Hygiene, vol. 84, pp. 386-389, 2011.

[13] C. Falade, O. Mokuolu, H. Okafor et al., "Epidemiology of congenital malaria in Nigeria: a multi-centre study," Tropical Medicine and International Health, vol. 12, no. 11, pp. 12791287, 2007.

[14] P. O. Obiajunwa, J. A. Owa, and O. O. Adeodu, "Prevalence of congenital malaria in Ile-Ife, Nigeria," Journal of Tropical Pediatrics, vol. 51, no. 4, pp. 219-222, 2005.

[15] A. D. Ekanem, M. U. Anah, and J. J. Udo, "The prevalence of congenital malaria among neonates with suspected sepsis in 
Calabar, Nigeria," Tropical Doctor, vol. 38, no. 2, pp. 73-76, 2008.

[16] M. K. Bouyou-Akotet, D. E. Ionete-Collard, M. MabikaManfoumbi et al., "Prevalence of Plasmodium falciparum infection in pregnant women in Gabon," Malaria Journal, vol. 2, no. 1, article 1, p. 18, 2003.

[17] O. J. Adebami, J. A. Owa, G. A. Oyedeji, O. A. Oyelami, and G. O. Omoniyi-Esan, "Associations between placental and cord blood malaria infection and fetal malnutrition in an area of malaria holoendemicity," American Journal of Tropical Medicine and Hygiene, vol. 77, no. 2, pp. 209-213, 2007.

[18] O. A. Idowu, C. F. Mafiana, and S. Dapo, "Malaria among pregnant women in Abeokuta, Nigeria," Tanzania Health Research Bulletin, vol. 8, no. 1, pp. 28-31, 2006. 


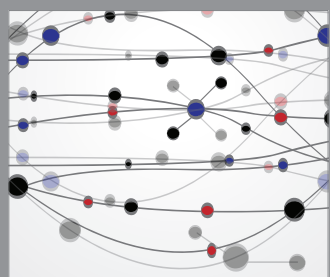

The Scientific World Journal
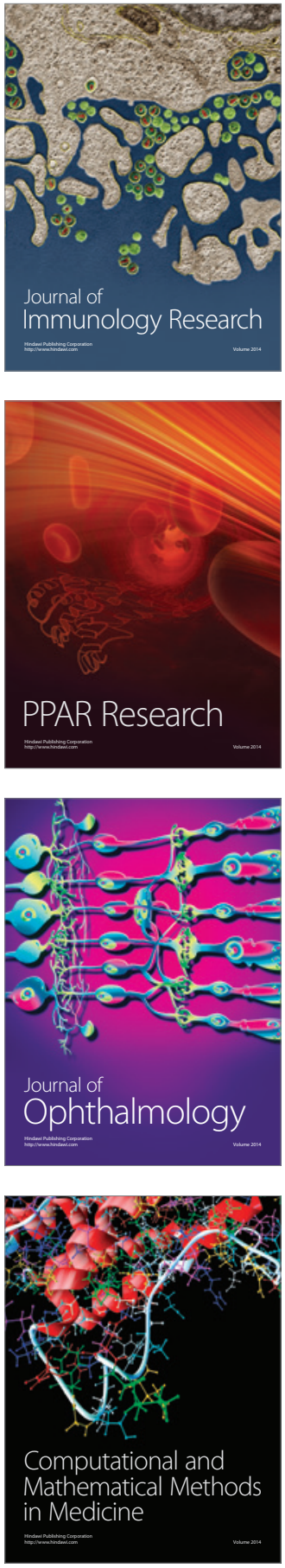

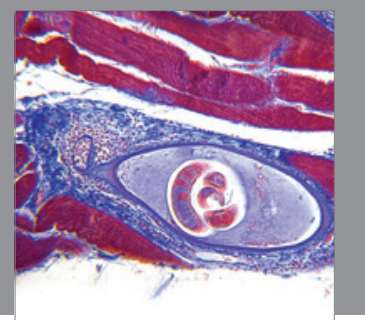

Gastroenterology

Research and Practice
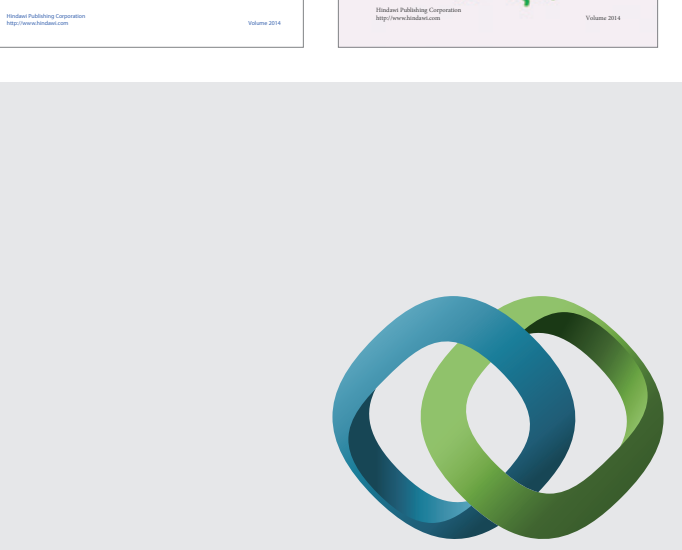

\section{Hindawi}

Submit your manuscripts at

http://www.hindawi.com
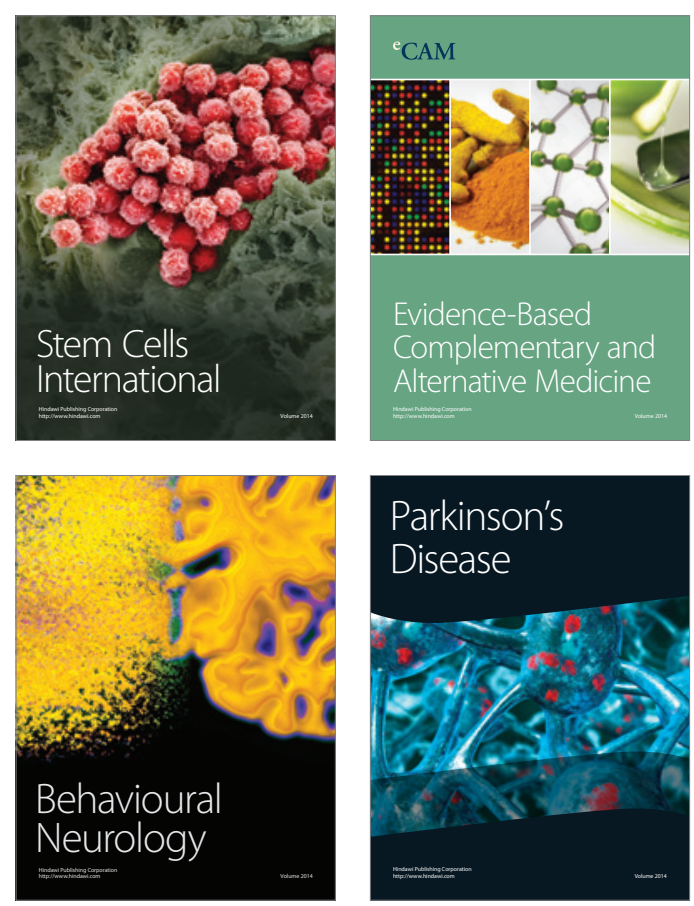

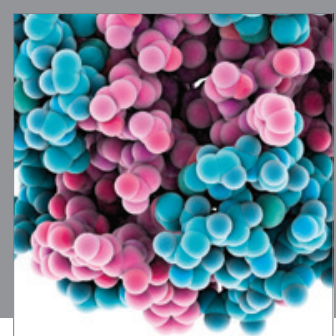

Journal of
Diabetes Research

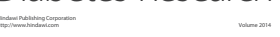

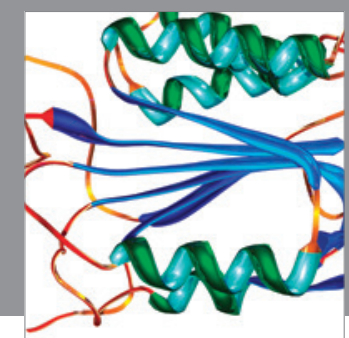

Disease Markers
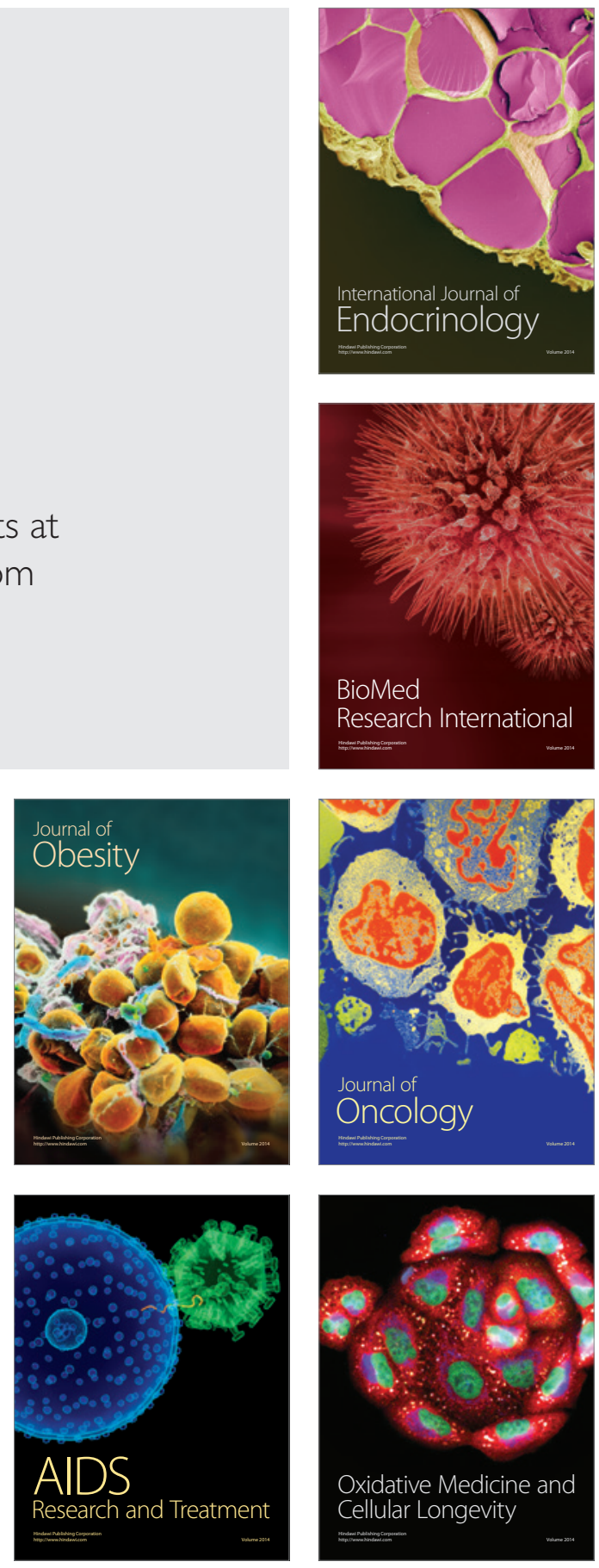